VOL. $10(1974), 95-105$.

\title{
Sabidussi-type theorems for stability
}

\section{Douglas D. Grant, D.A. Holton, and K.L. McAvaney}

\begin{abstract}
In this note we give details of a method by which we can produce an index-0 graph from any unstable graph and use it to show that given any finite group there exists an index-0 graph whose automorphism group is isomorphic, as an abstract group, to the given group. We proceed to construct two infinite families of connected index-0 graphs with connected complements whose automorphism group contains a transposition. This enables us to produce, for any finite group $G$, an index-0 graph whose automorphism group, isomorphic as an abstract group to $C_{2} \times G$, contains a transposition.
\end{abstract}

1. Index-0 graphs with given automorphism group

Throughout this note all graphs $G$ are undirected, have no loops or multiple edges, and have finite vertex set $V(G)$ with $|V(G)|=p$. The basic terminology and notation is that of Behzad and Chartrand, [1].

If $v \in V(G)$, then by $G_{v}$ we mean the induced subgraph $(V(G)-\{v\})$ of $G$, and by $G_{v_{1} v_{2}, \ldots, v_{k}}$ we mean $\left(\left[\ldots\left(\left(G_{v_{1}}\right)_{v_{2}}\right) \ldots\right)_{v_{k-1}}\right)_{v_{k}} \cdot G$ is said to be semi-stable (at $v \in V(G)$ ) if $\Gamma\left(G_{v}\right)=\Gamma(G)_{v}$, where $\Gamma(G)_{v}$ is the subgroup of $\Gamma(G)$ which fixes vertex $v$. If $G$ is a graph which is not semi-stable we call $G$ an index-0 graph (see [3]). If there exists a sequence $\left\{v_{1}, \ldots, v_{p}\right\}$ of all the vertices of $G$ such that $G$

Received 4 October 1973 . The first author's research was supported in part by a Commonwealth Postgraduate Research Award. 
is semi-stable at $v_{1}$ and $G_{v_{1}}, \ldots, v_{k}$ is semi-stable at $v_{k+1}$ for $1 \leq k \leq p-1$ we say that $G$ is stable.

We define the subgraph $G_{v}$ of $G$ to be l-admissible if and only if $G$ is semi-stable at $v$. We denote by $A_{l}(G)$ the collection $\left\{G_{v}: G\right.$ is semi-stable at $\left.v\right\}$, by $B_{1}(G)$ the set of distinct components of the graphs in $A_{1}(G)$ and by $C_{1}(G)$ the unstable graphs in $B_{1}(G)$ which are not components of $G$. If $M$ is a set of graphs define $A_{1}(M)$ as $\left\{A_{1}(G): G \in M\right\}$, and $B_{1}(M)$ as the set of distinct components of the graphs in $A_{1}(M)$. If $G$ is a graph, define $C_{0}(G)$ to be $G$ if $G$ is unstable and the empty graph if $G$ is stable. For $k=1,2, \ldots$, define $B_{k}(G)$ to be $B_{1}\left(C_{k-1}(G)\right)$ and $C_{k}(G)$ to be the set of unstable graphs in $B_{k}(G)$ which are not in $\bigcup_{i=0}^{k-1} C_{i}(G)$. Let $c(G)=\max \left\{k: C_{k}(G) \neq \emptyset\right\}$. Thus either each graph in $C_{c(G)}(G)$ is an index-0 graph or each unstable component of each 1-admissible subgraph of each graph in $C_{c(G)}(G)$ is in $\bigcup_{i=0}^{c(G)} C_{i}(G)$. Finally, we let $G^{0}=\bigcup_{i=0}^{c(G)} C_{i}(G)$.

In Figure 1 we illustrate the process of obtaining $G^{0}$ from $G$. LEMMA I ([4], Theorem 5). If $D=\left\{G^{1}, \ldots, G^{n}\right\}$, then $\bigcup_{i=1}^{n} G^{i}$ is stable if and only if each $G^{i}$ is stable.

LEMMA 2. Let $G$ be a graph. If $C_{k}(G) \neq \varnothing$, then some component of each groph in $A_{1}\left(C_{k-1}(G)\right)$ is in $\bigcup_{i=0}^{k} C_{i}(G)$.

Proof. By definition, no graph in $C_{k-1}(G)$ is stable. Thus no l-admissible subgraph of such a graph can be stable, whence at least one component of each 1-admissible subgraph of each graph in $C_{k-1}(G)$ is 

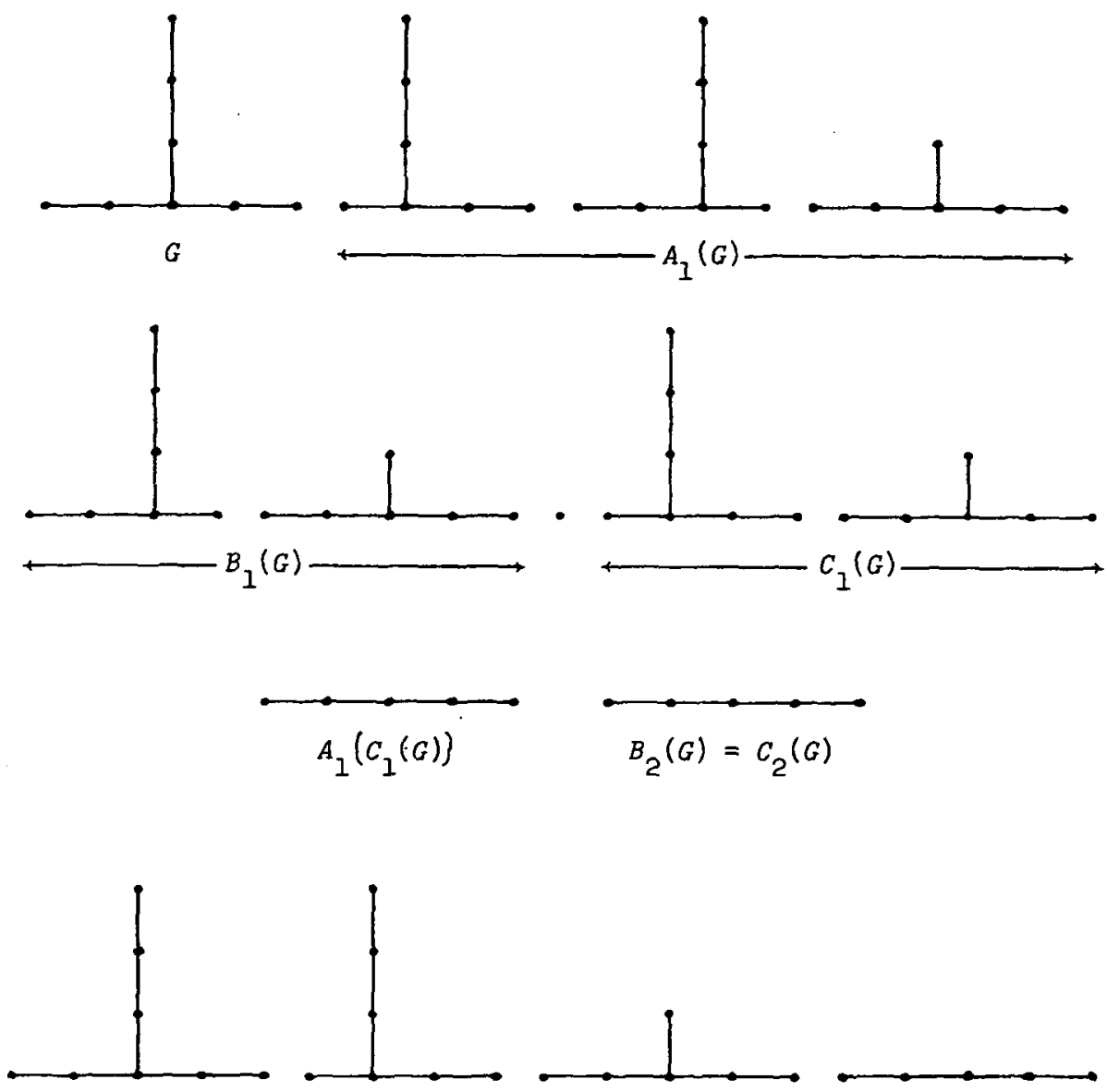

$G^{0}$

Figure 1

unstable. Such a component is, by definition, in $C_{k}(G)$ or $\bigcup_{i=0}^{k-1} C_{i}(G)$, whence the lemma follows.

The next result is Theorem 5 of [3]. To enable us to state it, we first need to introduce some notation.

If $D$ is a collection of graphs (not necessarily distinct) at least one of which is semi-stable, then $A_{1}(D) \& D$ means that no component of 
some graph in $A_{1}(D)$ is a component of a graph in $D$.

LEMMA 3. Let $D=\left\{G^{7}, \ldots, G^{n}\right\}$ be a collection of connected graphs at least one of which is semi-stable. Then $\bigcup_{i=1}^{n} G^{i}$ is semi-stable if and only if $A_{1}(D) \nless D$.

COROLLARY. Let $D$ as defined above be a collection of graphs (not necessarily connected) at least one of which is semi-stable. Let $D_{1}$ be the collection of components of the graphs in $D$. Then $\bigcup_{i=1}^{n} G^{i}$ is semi-stable if and only if $A_{1}\left(D_{1}\right) \nless D_{1}$.

We may now deduce:

THEOREM 1. If $G$ is an unstable groph, then $G^{0}$ is an index-0 graph.

Proof. We remark first of all that $G^{0}$ is empty if $G$ is stable. If $G$ is unstable as hypothesised, then either $G$ is an index-0 graph or $G$ is semi-stable. In the former case $G^{0}=G$ and the theorem is proved. If $G$ is semi-stable then it follows from Lemmas 2 and 3 and the definition of $G^{0}$ that $G^{0}$ is an index-0 graph.

In [2], Frucht proved:

LEMMA 4. Given any finite group $G$ there exists a groph $F(G)$ (the Frucht graph of $G$ ), whose automorphism group is isomorphic, as an abstract group, to $G$.

Frucht based his construction of $F(G)$ on the Cayley colour graph of $G$. From the construction also follows:

LEMMA 5. The automorphism group $\Gamma=\Gamma(F)$ of the Frucht graph of a group acts semi-regularly on $V(F)$ (that is, each non-identity element of $\Gamma$ is fixed-point free).

Sabidussi, [9], has extended Frucht's results, showing that, given any finite group, there exists a graph possessing various given graphtheoretical properties whose automorphism group is isomorphic, as an 
abstract group, to the given group. We now prove a Sabidussi-type theorem in which we prescribe our graphs to be index-0 graphs.

THEOREM 2. Let $G$ be any finite group of order $>1$. Then if $F$ is the Frucht groph of $G$, the graph $F^{0}$ is an index-0 groph whose automorphism group is isomorphic, as an abstract group, to $G$.

Proof. By Lemma 5, if $v$ is any vertex of $F, \Gamma(F)_{v}$, the set of elements in $\Gamma(F)$ which fix $v$, is the identity. Thus $\Gamma(F)$ contains no transpositions, for certainly $|V(F)|>2$. By Lemma 3 of [7], $F$ is unstable. From Theorem $I$ we deduce that $F^{0}$ is non-empty and is an index-0 graph. If $F$ is not semi-stable, then $F^{0}=F$ anò $\Gamma\left(F^{0}\right)=\Gamma(F) \cong G$. Thus assume that $F$ is semi-stable and let $v$ be an arbitrary vertex at which $F$ is semi-stable. It follows that $\Gamma\left(F_{v}\right)=\Gamma(F)_{v}$, and is the identity. Thus $F_{v}$ is asymmetric, whence each component of $F_{v}$ is asymmetric. We deduce that each graph in $A_{1}(F)$, $B_{1}(F)$ and $C_{1}(F)$ is asymetric. By continuing the above argument, noting that the automorphism group of each graph in $C_{1}(F)$, being the identity, is semi-regular, we conclude that each graph in $C_{k}(F)$, for $k=0, \ldots, c(F)$, is asymmetric. It then follows, since the components of $F^{0}$ are all different, that $\Gamma\left(F^{0}\right) \cong \Gamma(F) \cong G$.

COROLLARY. Given any finite group $G$ there exists an index-o graph whose automorphism growp is isomorphic, as an abstract group, to $G$.

Proof. If the order of $G$ is $>1$, the graph $F$ defined above is a suitable graph. If $G$ is the identity group, the tree $E_{7}$ of Figure 2 is a suitable graph.

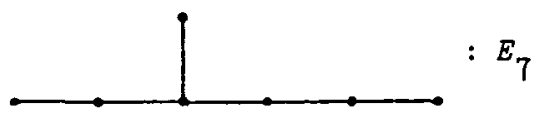

Figure 2 


\section{Index-0 graphs whose automorphism group contains a transposition}

Lemma 3 of [7] states that if $G$ is a stable graph then either $G$ is $K_{1}$ or $\Gamma(G)$ contains a transposition. A great deal of research (see [5], [6] and [8]) has been done on the problem of finding those graphs whose automorphism group contains a transposition which are stable. Not all such graphs are stable, as witnessed by the graph $G$ of Figure $3 . \Gamma(G)$ contains the transposition (12) but $G$ is unstable.

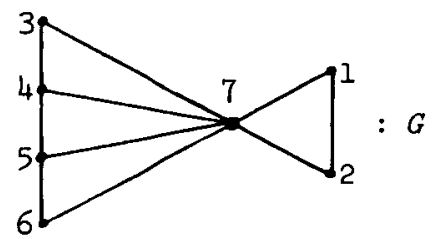

Figure 3

Here we show that in fact there exist graphs whose automorphism group contains a transposition which are not even semi-stable.

Let $G$ be a graph. Denote by $K^{\prime}(G)$ the graph $G+G=\overline{\bar{G} \cup \bar{G}}$ and by $D^{\prime}(G)$ the graph $G+G+G=\overline{\bar{G} \cup \bar{G} \cup \bar{G}}$. Define $K(G)$ and $D(G)$ to be the graphs shown in Figure 4, where the various symbols used have the indicated meanings. In Figure 5 we show $K\left(P_{4}\right)$ and $D\left(P_{4}\right)$ in full detail.

We now obtain, as abstract groups, the automorphism groups of $K(G)$ and $D(G)$.

THEOREM 3. FOr any graph $G$, $\Gamma(K(G)) \cong \Gamma(D(G)) \cong C_{2} \times \Gamma(G) \times \Gamma(G) \times \Gamma(G) \times \Gamma(G) \times \Gamma(G) \times \Gamma(G)$.

Proof. It is easy to see that the latter group is a subgroup of each of the automorphism groups. That there are no other automorphisms is proved by exhaustion; we omit the details.

For all $G, \Gamma(K(G))$ and $\Gamma(D(G))$ both contain a transposition. We now show that neither of these graphs is semi-stable if $G$ is not semistable. 
Sab dussi-type theorems

101

0

G

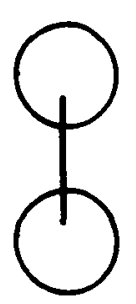

$K^{\prime}(G)$

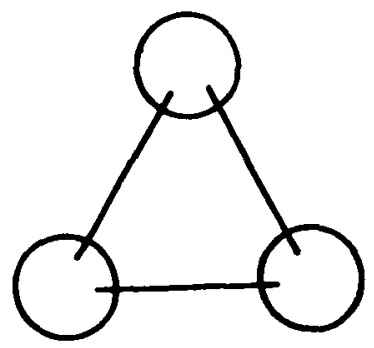

$D^{\prime}(G)$

$$
K_{1}+G=\overline{\overline{K_{1}} \cup \bar{G}}
$$
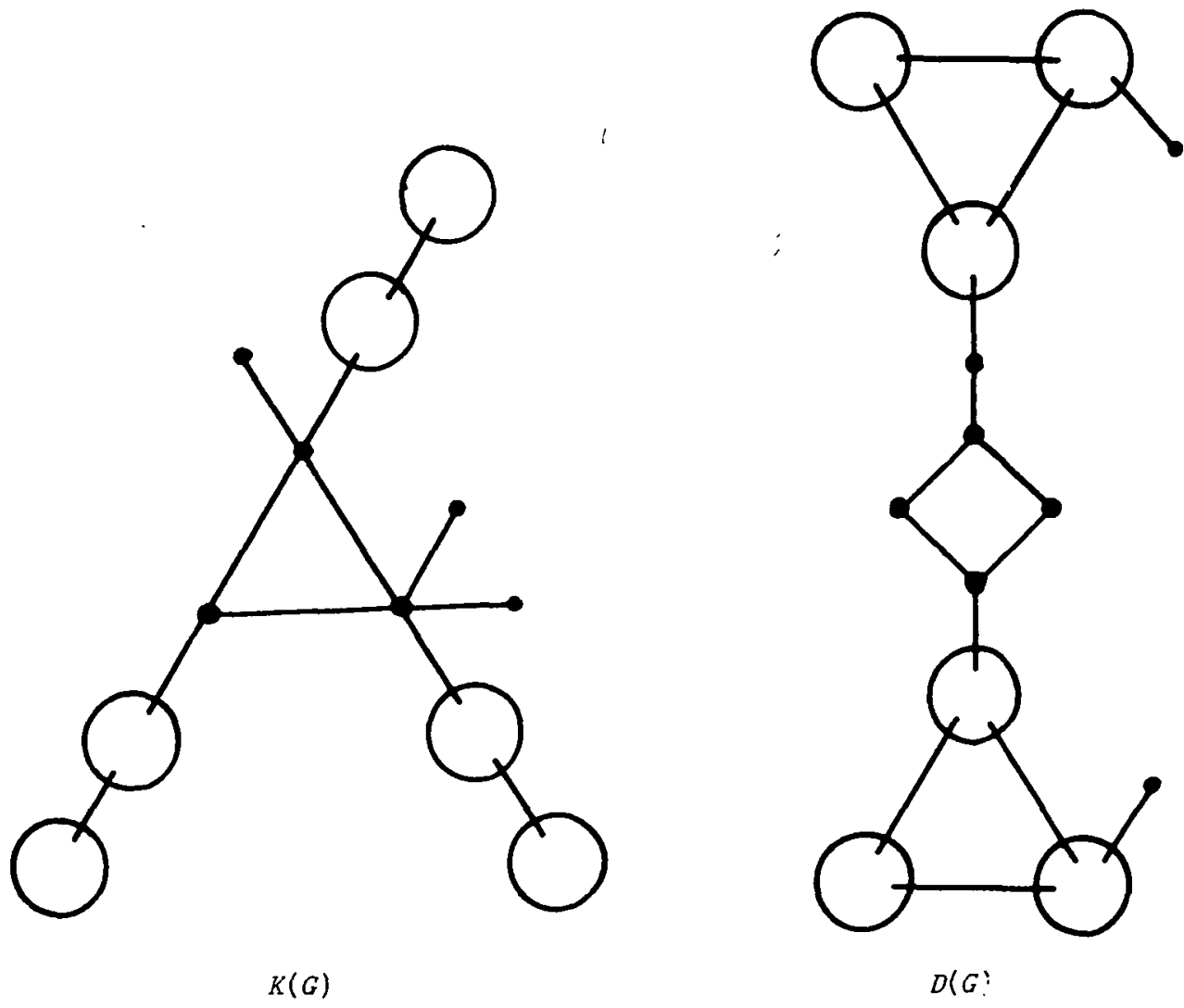

Figure 4

https://doi.org/10.1017/S0004972700040685 Published online by Cambridge University Press 

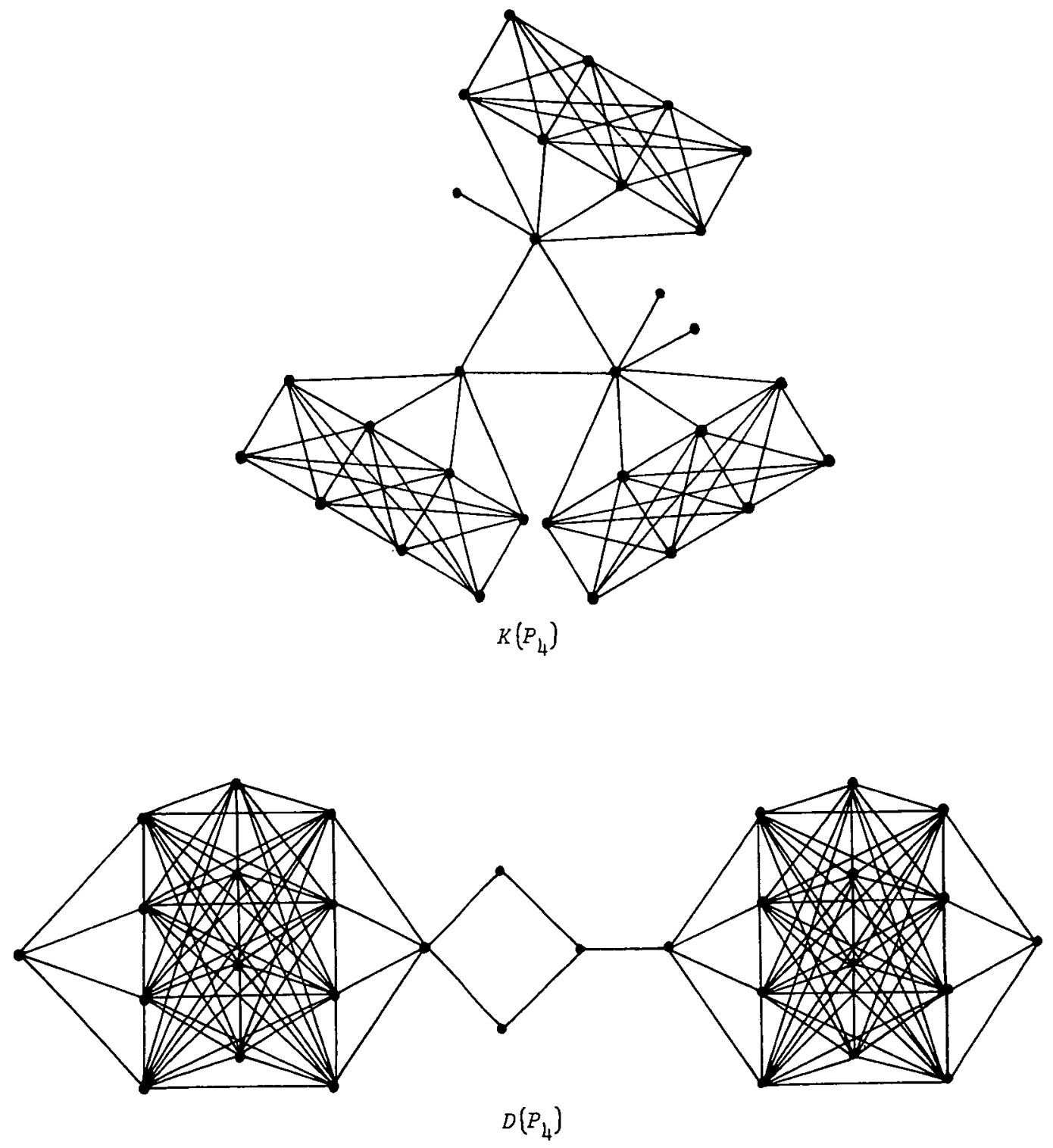

Figure 5 
THEOREM 4. If $G$ is an index-0 groph, then $K(G)$ and $D(G)$ are both index-0 grophs.

Proof. We give here a proof for $K(G)$; that for $D(G)$ is similar. Let the vertices of $G$ be labelled $w_{l}, \ldots, w_{r}$, and those of $K(G)$ all:ading to the scheme illustrated in Figure 6 , where the vertices in copy $i$ of $G$ are labelled $v_{i j}, j=1, \ldots, r$, corresponding respectively to $w_{j}, j=l, \ldots, r$.

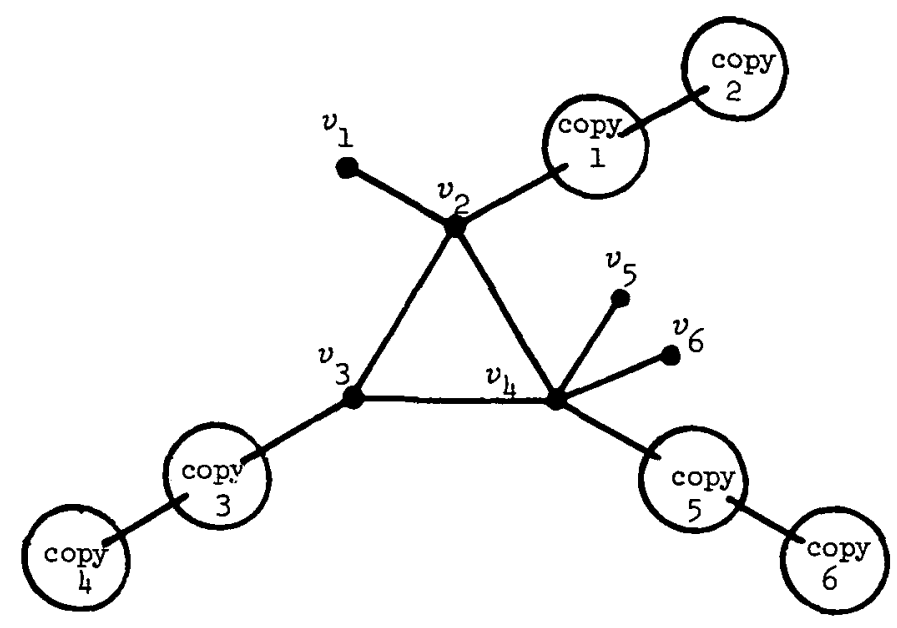

Figure 6

Removal of $v_{1}$ from $K=K(G)$ introduces automorphisms mapping $v_{2}$ onto $v_{3}$ and vertices in copies 1 and 2 of $G$ onto vertices in copies 3 and 4 respectively. Removal of $v_{5}$ or $v_{6}$ introduces automorphisms mapping $v_{2}$ onto $v_{4}$ and vertices in copies 1 and 2 of $G$ onto vertices in copies 5 and 6 respectively. Removal of $v_{2}, v_{3}$ or $v_{4}$ introduce respectively automorphisms mapping vertices in copy $I$ of $G$ onto vertices in copy 2, vertices in copy 3 onto vertices in copy 4 or vertices in copy 5 onto vertices in copy 6 . Removal of vertex $v_{i j}$ introduces the automorphism $a$ given by $a\left(v_{i k}\right)=v_{i s(k)}$ for $k \neq j$, which is generated 
by the automorphism $a^{\prime}$ which is introduced in $\Gamma\left(G_{w_{j}}\right)$ by removing vertex $w_{j}$ from $G$, this automorphism being given by $a^{\prime}\left(w_{k}\right)=w_{s(k)}$ for $k \neq j$. Thus removal of any vertẹx from $K$ introduces new automorphisms, whence $\Gamma\left(K_{v}\right) \neq \Gamma(K)_{v}$ for any $v \in V(K)$, whence $K$ is an index-0 graph.

Theorem 4 thus provides us with two infinite families of index-0 graphs whose automorphism group contains a transposition. We remark also that for all $G$, both $K(G)$ and $D(G)$ are complement connected (that is, $K(G), \overline{K(G)}, D(G)$ and $\overline{D(G)}$ are all connected).

The smallest (in the sense of having the least number of vertices) index- 0 graph whose automorphism group contains a transposition, which is given by Theorem 4, is $K\left(P_{4}\right)$, this graph having 30 vertices. We conjecture that in fact this graph is the smallest index-0 graph whose automorphism group contains a transposition.

We conclude by observing:

THEOREM 5. Given any finite group $G$ there exists an index-0 graph whose automorphism group contains a transposition and which is isomorphic, as an abstract group, to $C_{2} \times G$.

Proof. Let $F$ be the Frucht graph of $G$. Then the graph $F^{\prime}=F^{0} \cup K\left(E_{7}\right)$ is an index-0 graph, being the union of two index-0 graphs, provided that $G$ is of order $>1$. If $G$ is the identity group, the graph $F^{\prime}=E_{7} \cup K\left(E_{7}\right)$ is an index-0 graph. In each case $F^{\prime}$ has automorphism group isomorphic to $C_{2} \times G$, as each component of $F^{\prime}$ is different.

\section{References}

[1] Mehdl Behzad and Gary Chartrand, Introduction to the theory of graphs (Allyn and Bacon, Boston, 1971).

[2] R. Frucht, "Herstellung von Graphen mit vorgegebener abstrakter Gruppe", Compositio Math. 6 (1939), 239-250. 
[3] Douglas D. Grant, "The stability index of graphs", Proc. Second Austral. Conf. Combinatorial Mathematics (Lecture Notes in Mathematics. Springer-Verlag, to appear).

[4] D.A. Holton, "A report on stable graphs", J. Austral. Math. Soc. 15 (1973), 163-171.

[5] D.A. Holton, "Stable trees", J. Austral. Math. Soc. 15 (1973), 476-481

[6] D.A. Holton and Douglas D. Grant, "Regular graphs and stability", submitted.

[1] D.A. Holton and Douglas D. Grant, "Products of trees and stability", submitted.

[8] K.L. McAvaney, Douglas D. Grant, D.A. Holton, "Stable and semi-stable uncyclic graphs", submitted.

[9] G. Sabidussi, "Graphs with given group and given graph-theoretical properties", Canad. J. Math. 9 (1957), 515-525.

Department of Mathematics,

University of Melbourne,

Parkville,

victoria. 